\title{
Periodic Cohomology Theories Defined by Elliptic Curves
}

\author{
PETER S. LANDWEBER, DOUGLAS C. \\ RAVENEL AND ROBERT E. STONG
}

\begin{abstract}
We use bordism theory to construct periodic cohomology theories, which we call elliptic cohomology, for which the cohomology of a point is a ring of modular functions. These are complex-oriented multiplicative cohomology theories, with formal groups associated to the universal elliptic genus studied by a number of authors $([\mathbf{C C}, \mathbf{L S}, \mathbf{O}, \mathbf{W} \mathbf{1}, \mathbf{Z}])$. We are unable to find a geometric description for these theories.
\end{abstract}

\section{Introduction}

One can argue that the cohomology theories of greatest importance in algebraic topology are cobordism theories, K-theories and ordinary cohomology theories; each class has a wide usefulness and its own geometric character. We shall present evidence for a fourth class of cohomology theories, which might be called elliptic cohomology theories, since they arise in connection with elliptic curves and (elliptic) modular forms. They are periodic theories, with two different periodicity elements of dimension 8 , whose coefficient rings (the cohomology of a point) can be naturally identified with rings of modular functions. In addition, they are complex-oriented with formal groups of the type arising from elliptic curves.

Although we shall produce such theories by two methods, we are unable to provide an intrinsic geometric definition. This glaring omission presents the fascinating problem of finding or constructing the appropriate objects and formalism to give a natural description of these cohomology theories. Work by

1991 Mathematics Subject Classification. Primary 55N22, 57R75, 57R77; Secondary 11F11, 14H52, 14L05.

The authors thank the National Science Foundation for financial support.

This paper is in final form and no version of it will be submitted for publication elsewhere. 
E. Witten $([\mathbf{W} \mathbf{1}, \mathbf{W} \mathbf{2}])$ and C. Taubes ([Ta]) leads one to suspect that quantum field theory and Dirac operators on free loop spaces will play a major role.

We shall view cohomology and homology as two sides of the same coin, since both can be identified with their representing spectra. Indeed, our methods are better suited to the construction of homology theories.

The coefficient rings and formal groups, for the theories of greatest interest to us, take the following form. Let $\delta$ and $\varepsilon$ be indeterminates of weights 2 and 4 , respectively, and introduce the graded polynomial ring

$$
\mathrm{M}_{*}=\mathbf{Z}\left[\frac{1}{2}\right][\delta, \varepsilon] .
$$

(The choice of symbol is meant to indicate "modular forms", in agreement with Theorem 1.5 and $\S 5$ below. An algebraic topologist would assign degree $2 k$ to an element of weight $k$.) Introduce the differential

$$
\omega=\left(1-2 \delta x^{2}+\varepsilon x^{4}\right)^{-\frac{1}{2}} d x=R(x)^{-\frac{1}{2}} d x
$$

on the Jacobi quartic

$$
y^{2}=1-2 \delta x^{2}+\varepsilon x^{4},
$$

and the corresponding logarithm

$$
g(x)=\int_{0}^{x}\left(1-2 \delta t^{2}+\varepsilon t^{4}\right)^{-\frac{1}{2}} d t \in \mathrm{M}_{*} \otimes \mathbf{Q}[[x]] .
$$

A formal group over $\mathrm{M}_{*} \otimes \mathbf{Q}$ is defined by

$$
F_{E}\left(x_{1}, x_{2}\right)=g^{-1}\left(g\left(x_{1}\right)+g\left(x_{2}\right)\right) ;
$$

indeed its coefficients all lie in $\mathrm{M}_{*}$ (see $\S 2$ ), and one has Euler's explicit formula ([E, O, L3 (appendix)]):

$$
F_{E}\left(x_{1}, x_{2}\right)=\frac{x_{1} \sqrt{R\left(x_{2}\right)}+x_{2} \sqrt{R\left(x_{1}\right)}}{1-\varepsilon x_{1}^{2} x_{2}^{2}} .
$$

The appropriate discriminant, of weight 12 , is

$$
\Delta=\varepsilon\left(\delta^{2}-\varepsilon\right)^{2} .
$$

The periodic cohomology theories are multiplicative, having as coefficient rings the localizations

$$
\mathrm{M}_{*}\left[\Delta^{-1}\right], \mathrm{M}_{*}\left[\varepsilon^{-1}\right], \mathrm{M}_{*}\left[\left(\delta^{2}-\varepsilon\right)^{-1}\right] .
$$

We also define a ring homomorphism on the oriented bordism ring $\Omega_{*}^{\mathrm{SO}}$,

$$
\varphi: \Omega_{*}^{\mathrm{SO}} \rightarrow \mathrm{M}_{*} \otimes \mathbf{Q},
$$

by the requirement that

$$
\omega=\left(\sum_{n \geq 0} \varphi\left(\mathbf{C P}^{2 n}\right) x^{2 n}\right) d x,
$$


noting that $\Omega_{*}^{\mathrm{SO}} \otimes \mathbf{Q}$ is a polynomial ring on the bordism classes $\left[\mathbf{C P}^{2 n}\right], n \geq 1$ ([Th, MS $]$ ). Indeed this universal elliptic genus maps $\Omega_{*}^{\mathrm{SO}}$ into $\mathrm{M}_{*}$ (see $\S 2$ ).

Let $\Omega_{*}^{\mathrm{SO}}(X)$ denote the oriented bordism of the space $X$. We shall apply the exact functor theorem $([\mathbf{L} \mathbf{1}])$, together with information about formal groups of elliptic curves ([L3]), to prove:

THEOREM 1.2. The functors

$$
\begin{aligned}
& \Omega_{*}^{\mathrm{SO}}(\cdot) \otimes_{\Omega_{*}^{\mathrm{SO}}} \mathrm{M}_{*}\left[\Delta^{-1}\right], \\
& \Omega_{*}^{\mathrm{SO}}(\cdot) \otimes_{\Omega_{*}^{\mathrm{SO}}} \mathrm{M}_{*}\left[\varepsilon^{-1}\right], \\
& \Omega_{*}^{\mathrm{SO}}(\cdot) \otimes_{\Omega_{*}^{\mathrm{SO}}} \mathrm{M}_{*}\left[\left(\delta^{2}-\varepsilon\right)^{-1}\right]
\end{aligned}
$$

are multiplicative homology theories, with coefficient rings (1.1) viewed as $\Omega_{*}^{\mathrm{SO}}$ modules via the elliptic genus $\varphi$. The formal groups of the corresponding cohomology theories are the canonical extensions of the formal group $F_{E}\left(x_{1}, x_{2}\right)$ over $\mathrm{M}_{*}$ described above.

We are also able to produce a homology theory with coefficient ring $\mathrm{M}_{*}$, by the method of bordism with singularities due to Sullivan and Baas ([B]). Since we have inverted 2 , this is a multiplicative homology theory $([\mathbf{M r}])$ which we denote by $\mathrm{M}_{*}(X)$ for a space $X$. There is a natural transformation

$$
\Phi: \Omega_{*}^{\mathrm{SO}}(\cdot) \rightarrow \mathrm{M}_{*}(\cdot),
$$

extending the elliptic genus $\varphi: \Omega_{*}^{\mathrm{SO}} \rightarrow \mathrm{M}_{*}$. One can obtain further homology theories

$$
\mathrm{M}_{*}(\cdot)\left[\Delta^{-1}\right], \mathrm{M}_{*}(\cdot)\left[\varepsilon^{-1}\right], \mathrm{M}_{*}(\cdot)\left[\left(\delta^{2}-\varepsilon\right)^{-1}\right]
$$

by localization. The point of Theorem 1.2, now, is that one has "Conner-Floyd theorems" (see $[\mathbf{C F 2}]$ ) for these periodic homology theories. That is, $\Phi$ induces isomorphisms

$$
\begin{aligned}
\Omega_{*}^{\mathrm{SO}}(\cdot) \otimes_{\Omega_{*}^{\mathrm{SO}}} \mathrm{M}_{*}\left[\Delta^{-1}\right] & \stackrel{\sim}{\longrightarrow} \mathrm{M}_{*}(\cdot)\left[\Delta^{-1}\right], \\
\Omega_{*}^{\mathrm{SO}}(\cdot) \otimes_{\Omega_{*}^{\mathrm{SO}}} \mathrm{M}_{*}\left[\varepsilon^{-1}\right] & \stackrel{\sim}{\longrightarrow} \mathrm{M}_{*}(\cdot)\left[\varepsilon^{-1}\right], \\
\Omega_{*}^{\mathrm{SO}}(\cdot) \otimes_{\Omega_{*}^{\mathrm{SO}}} \mathrm{M}_{*}\left[\left(\delta^{2}-\varepsilon\right)^{-1}\right] & \stackrel{\sim}{\longrightarrow} \mathrm{M}_{*}(\cdot)\left[\left(\delta^{2}-\varepsilon\right)^{-1}\right] .
\end{aligned}
$$

The periodic homology theories appear to have a greater claim to one's attention than does the connective theory $\mathrm{M}_{*}(\cdot)$. For example, we shall show that the theories with coefficient rings $\mathrm{M}_{*}\left[\varepsilon^{-1}\right]$ and $\mathrm{M}_{*}\left[\left(\delta^{2}-\varepsilon\right)^{-1}\right]$ map naturally to KO-theory (with 2 inverted). We refer to all the periodic theories introduced here as elliptic homology (or cohomology).

The same method entitles us to construct multiplicative homology theories with coefficient rings

and

$$
\mathbf{Z}\left[\frac{1}{6}\right]\left[g_{2}, g_{3}, \Delta^{-1}\right]
$$

$$
\mathbf{Z}\left[a_{1}, a_{2}, a_{3}, a_{4}, a_{6}, \Delta^{-1}\right]
$$


corresponding to the Weierstrass equations

$$
y^{2}=4 x^{3}-g_{2} x-g_{3}
$$

and

$$
y^{2}+a_{1} x y+a_{3} y=x^{3}+a_{2} x^{2}+a_{4} x+a_{6}
$$

for elliptic curves. Here the $a_{i}$ are indeterminates with wt $a_{i}=i$; similarly, $g_{2}$ and $g_{3}$ are indeterminates with weights 4 and 6 , respectively. We refer to Silverman $[\mathbf{S i}]$ for standard notions and facts about elliptic curves. For example, see [Si, p. 46] for the discriminant $\Delta=\Delta\left(a_{1}, \ldots, a_{6}\right)$; the classical formula in terms of $g_{2}$ and $g_{3}$ is $\Delta=g_{2}{ }^{3}-27 g_{3}{ }^{2}$. The formal group is constructed in [ $\mathbf{S i}$, Ch. IV], and gives a universal expression for the addition on the elliptic curve near the origin in terms of a suitable uniformizing parameter.

Notice that we are forced to invert 2 when constructing the homology theories of greatest interest. We of course have the desire to retain 2-primary information, which is often of paramount importance in topology. By replacing $\Omega_{*}^{\mathrm{SO}}(\cdot)$ with complex bordism $\Omega_{*}^{\mathrm{U}}(\cdot)$, and using either the universal Weierstrass equation (1.4) as above, or instead a homogeneous Deuring normal form ([Si, p. 327])

$$
y^{2}+\alpha x y+\beta y=x^{3}
$$

with $\Delta=\beta^{3}\left(\alpha^{3}-27 \beta\right)$, we can retain 2-primary information but lose contact with topological applications.

It is well-known that the coefficients $g_{2}$ and $g_{3}$ in the Weierstrass equation (1.3) can be interpreted as modular forms for $\mathrm{SL}_{2}(\mathbf{Z})([\mathbf{K}, \mathbf{S e}])$. As a warmup for the proof of Theorem 1.5, one verifies easily that

$$
\mathbf{Z}\left[\frac{1}{6}\right]\left[g_{2}, g_{3}, \Delta^{-1}\right]
$$

can be identified with the ring of modular functions for $\mathrm{SL}_{2}(\mathbf{Z})$ which are holomorphic on the upper half-plane $\mathcal{H}$ and whose $q$-expansions have all coefficients in $\mathbf{Z}\left[\frac{1}{6}\right]$.

Returning to the ring $\mathrm{M}_{*}=\mathbf{Z}\left[\frac{1}{2}\right][\delta, \varepsilon]$ of polynomials in the coefficients of the Jacobi quartic

$$
y^{2}=1-2 \delta x^{2}+\varepsilon x^{4},
$$

we shall interpret $\mathrm{M}_{*}$ and its localizations (1.1) as rings of modular functions for the subgroup $\Gamma_{0}(2)$ of the modular group $\mathrm{SL}_{2}(\mathbf{Z})$ consisting of all matrices $\left(\begin{array}{ll}a & b \\ c & d\end{array}\right)$ in $\mathrm{SL}_{2}(\mathbf{Z})$ with $c$ even. $\Gamma_{0}(2)$ is a non-normal subgroup of index 3 in $\mathrm{SL}_{2}(\mathbf{Z})$, with a fundamental domain

$$
\left\{z: \operatorname{Im} z>0,0<\operatorname{Re} z<1,\left|z-\frac{1}{2}\right|>\frac{1}{2}\right\}
$$

for its action on the upper half-plane $\mathcal{H}$, and the two cusps $0, \infty$. Let $\mathrm{M}_{*}\left(\Gamma_{0}(2)\right)$ denote the ring of modular forms for $\Gamma_{0}(2)$, as defined in $\S 5$. 
THEOREM 1.5. $\mathbf{C}[\delta, \varepsilon]$ is naturally isomorphic to the ring $\mathrm{M}_{*}\left(\Gamma_{0}(2)\right)$ of modular forms for $\Gamma_{0}(2)$, with $\delta$ and $\varepsilon$ having weights 2 and 4 , respectively. This isomorphism sends the subring $\mathrm{M}_{*}=\mathbf{Z}\left[\frac{1}{2}\right][\delta, \varepsilon]$ to the modular forms whose $q$-expansions at the cusp $\tau=\infty$ have coefficients in $\mathbf{Z}\left[\frac{1}{2}\right]$. Moreover, the localizations $\mathrm{M}_{*}\left[\Delta^{-1}\right], \mathrm{M}_{*}\left[\varepsilon^{-1}\right]$ and $\mathrm{M}_{*}\left[\left(\delta^{2}-\varepsilon\right)^{-1}\right]$ correspond to the rings of modular functions which are holomorphic on $\mathcal{H}, \mathcal{H} \cup\{0\}$ and $\mathcal{H} \cup\{\infty\}$, respectively, and whose q-expansions have coefficients in $\mathbf{Z}\left[\frac{1}{2}\right]$.

The rest of this paper is organized as follows. We discuss elliptic genera in $\S 2$, and then apply the technique of bordism with singularities in $\S 3$ to construct a homology theory with coefficient ring $\mathrm{M}_{*}=\mathbf{Z}\left[\frac{1}{2}\right][\delta, \varepsilon]$. We apply the exact functor theorem to prove Theorem 1.2 in $\S 4$, and examine the connection with KO-theory there; we construct several additional periodic homology theories by the same method. In $\S 5$ we identify the coefficient rings of the periodic homology theories with rings of modular functions.

The results of this paper have been announced, with brief indication of proofs, in $[\mathbf{L 2}]$.

The authors are grateful to David and Gregory Chudnovsky, Serge Ochanine and Don Zagier for advice, and to Haynes Miller for his encouragement.

This paper dates from 1987, when this subject was new, and does not take into account later contributions to the field. We apologize for the long delay in publication, and wish to call attention to Jens Franke's excellent treatment of these topics, with notable simplifications and clarifications, in his paper $O n$ the construction of elliptic cohomology $[\mathbf{F}]$. In particular, Franke proves that in Theorem 1.2 one can invert any homogeneous element of positive degree.

In addition, a construction of "integral" elliptic homology based on Spin bordism has been given by Matthias Kreck and Stephan Stolz $[\mathbf{K S}]$, and has been further clarified by Mark Hovey [Ho]. This theory does not require that one invert 2, and is based on a refined elliptic genus defined by Serge Ochanine [O1], which is sensitive to 2-primary phenomena in dimensions $8 k+1$ and $8 k+2$.

\section{Elliptic genera}

We need to develop some preliminaries on bordism theory, formal groups and elliptic genera.

2.1. The oriented bordism ring $\Omega_{*}^{\mathrm{SO}}$ is obtained from closed smooth oriented manifolds under the relation of bordism ([Th, MS $]$ ). One knows that

$$
\Omega_{*}^{\mathrm{SO}} \otimes \mathbf{Q}=\mathbf{Q}\left[x_{4}, x_{8}, \ldots\right]
$$

with algebraically independent polynomial generators which may be taken to be the bordism classes $x_{4 n}=\left[\mathrm{CP}^{2 n}\right], n \geq 1$. By a multiplicative genus, in the sense of Hirzebruch $[\mathbf{H}]$, is meant a ring homomorphism

$$
\varphi: \Omega_{*}^{\mathrm{SO}} \rightarrow R
$$


to a commutative algebra over $\mathbf{Q}$, with $\varphi(1)=1$. The best known examples of multiplicative genera are the signature and the Â-genus. Evidently, a multiplicative genus is uniquely specified by its logarithm

$$
g(x)=\sum_{n \geq 0} \frac{\varphi\left(\mathbf{C P}^{2 n}\right)}{2 n+1} x^{2 n+1},
$$

which can be any odd series in $R[[x]]$ with linear term $x$.

2.2. By an elliptic genus $([\mathbf{O}])$ is meant a multiplicative genus $\varphi$ for which there are elements $\delta, \varepsilon \in R$ so that the logarithm $g(x)$ of $\varphi$ has the form

$$
g(x)=\int_{0}^{x}\left(1-2 \delta t^{2}+\varepsilon t^{4}\right)^{-\frac{1}{2}} d t .
$$

Then $\delta=\varphi\left(\mathbf{C P}^{2}\right)$ and $\varepsilon=\varphi\left(\mathbf{H P}^{2}\right)$. One obtains the signature by taking $\delta=\varepsilon=1$, and the Â-genus by choosing $\delta=-\frac{1}{8}$ and $\varepsilon=0$. One calls the elliptic genus $\varphi: \Omega_{*}^{\mathrm{SO}} \rightarrow R$ universal if $\delta$ and $\varepsilon$ are algebraically independent over $\mathbf{Q}$ and $R=\mathbf{Q}[\delta, \varepsilon]$.

To any multiplicative genus $\varphi: \Omega_{*}^{\mathrm{SO}} \rightarrow R$, one associates a formal group

$$
F(x, y)=g^{-1}(g(x)+g(y)),
$$

where $g(x)$ denotes the logarithm of $\varphi$ and $g^{-1}(u)$ is the inverse under composition. The formal group associated to an elliptic genus $\varphi$, with parameters $\delta, \varepsilon \in R$ and

$$
R(x)=1-2 \delta x^{2}+\varepsilon x^{4},
$$

has the form

$$
F_{E}(x, y)=\frac{x \sqrt{R(y)}+y \sqrt{R(x)}}{1-\varepsilon x^{2} y^{2}}
$$

this is Euler's expression for the addition formula for the elliptic integral

$$
\int_{0}^{x} R(t)^{-\frac{1}{2}} d t
$$

([E, O, L3 (appendix)]).

2.3. Similarly, the complex bordism ring $\Omega_{*}^{\mathrm{U}}$ is obtained from closed smooth manifolds whose stable tangent bundles have complex structures. One has

$$
\Omega_{*}^{\mathrm{U}} \otimes \mathbf{Q}=\mathbf{Q}\left[y_{2}, y_{4}, \ldots\right]
$$

with algebraically independent polynomial generators $y_{2 n}=\left[\mathrm{CP}^{n}\right], n \geq 1$. If $R$ is a commutative ring with unit, then a formal group over $R$ is a power series

$$
F(x, y) \in R[[x, y]]
$$


satisfying the identities

$$
\left\{\begin{array}{l}
F(x, 0)=F(0, x)=x \\
F(x, F(y, z))=F(F(x, y), z) \\
F(x, y)=F(y, x)
\end{array}\right.
$$

In case $R$ is a $\mathbf{Q}$-algebra and $\varphi: \Omega_{*}^{\mathrm{U}} \rightarrow R$ is a ring homomorphism, we define a logarithm

and a formal group

$$
g(x)=\sum_{n \geq 0} \frac{\varphi\left(\mathbf{C P}^{n}\right)}{n+1} x^{n+1}
$$

$$
F(x, y)=g^{-1}(g(x)+g(y))
$$

When $R=\Omega_{*}^{\mathrm{U}} \otimes \mathbf{Q}$ and $\varphi$ is the inclusion of $\Omega_{*}^{\mathrm{U}}$ into $\Omega_{*}^{\mathrm{U}} \otimes \mathbf{Q}\left(\Omega_{*}^{\mathrm{U}}\right.$ has no torsion $)$ we write $F_{U}(x, y)$ for the resulting formal group. We refer to $[\mathbf{R}$, Appendix 2] for the following result, due to Mischenko and Quillen [Q].

THEOREM 2.4. The formal group $F_{U}(x, y)$ is a formal group over $\Omega_{*}^{\mathrm{U}}$, whose coefficients generate $\Omega_{*}^{\mathrm{U}}$. Moreover, $F_{U}$ is a universal formal group: for any formal group $F$ over $R$, there is a unique homomorphism $\varphi: \Omega_{*}^{\mathrm{U}} \rightarrow R$ so that $F$ is obtained from $F_{U}$ by applying $\varphi$ to all coefficients.

2.5. We return to Euler's formal group $F_{E}(x, y)$ associated to an elliptic genus $\varphi: \Omega_{*}^{\mathrm{SO}} \rightarrow R$ with parameters $\delta, \varepsilon \in R$. It is evident that $\varphi$ maps $\Omega_{*}^{\mathrm{SO}}$ into $\mathbf{Q}[\delta, \varepsilon]$, but we can make a sharper statement:

Proposition 2.6. The elliptic genus $\varphi$ maps $\Omega_{*}^{\mathrm{SO}}$ into $\mathbf{Z}\left[\frac{1}{2}\right][\delta, \varepsilon]$.

At the same time we will prove

Proposition 2.7. The formal group $F_{E}(x, y)$ associated to the elliptic genus $\varphi$ is defined over the subring $\mathbf{Z}\left[\frac{1}{2}\right][\delta, \varepsilon]$ of $R$.

PRoOFs. Making use of the binomial expansion of

$$
\begin{aligned}
R(x)^{-\frac{1}{2}} & =\left(1-2 \delta x^{2}+\varepsilon x^{4}\right)^{-\frac{1}{2}} \\
& =\sum_{n \geq 0} P_{n}(\delta, \varepsilon) x^{2 n},
\end{aligned}
$$

one sees easily that all coefficients lie in $\mathbf{Z}\left[\frac{1}{2}\right][\delta, \varepsilon]$; in fact they become the wellknown Legendre polynomials $P_{n}(\delta)$ when $\varepsilon$ is put equal to 1 . From this, it is clear that all coefficients of $\sqrt{R(x)}$ also lie in $\mathbf{Z}\left[\frac{1}{2}\right][\delta, \varepsilon]$. The explicit form of Euler's formal group now yields Proposition 2.7.

In addition to $\varphi: \Omega_{*}^{\mathrm{SO}} \rightarrow R$, we consider the composition

$$
\Omega_{*}^{\mathrm{U}} \rightarrow \Omega_{*}^{\mathrm{SO}} \stackrel{\varphi}{\longrightarrow} R \text {. }
$$

One knows ([St, p. 180]) that the forgetful homomorphism $\Omega_{*}^{\mathrm{U}} \rightarrow \Omega_{*}^{\mathrm{SO}}$ is onto modulo torsion. Since $R$ is assumed to be a $\mathbf{Q}$-algebra and so has no torsion, 
$\varphi\left(\Omega_{*}^{\mathrm{SO}}\right)$ is equal to the image of $\Omega_{*}^{\mathrm{U}}$ in $R$. The composition $\Omega_{*}^{\mathrm{U}} \rightarrow R$ classifies the formal group over $R$, so its image is the subring of $R$ generated by the coefficients of the formal group, in view of Theorem 2.4. Applying Proposition 2.7 , we conclude that $\mathbf{Z}\left[\frac{1}{2}\right][\delta, \varepsilon]$ contains this subring.

REMARK 2.8. With greater effort, one can determine the images of $\Omega_{*}^{\mathrm{SO}}$ and the Spin bordism ring $\Omega_{*}^{\text {Spin }}$ in $\mathbf{Z}\left[\frac{1}{2}\right][\delta, \varepsilon]$, for any elliptic genus. See $[\mathbf{L 2}, \S 5]$ and [CCLOS].

Remark 2.9. Proposition 2.6 permits us to view a univeral elliptic genus as mapping to $\mathbf{Z}\left[\frac{1}{2}\right][\delta, \varepsilon]$ with $\delta$ and $\varepsilon$ algebraically independent.

\section{Bordism with singularities}

3.1. The oriented bordism groups of a space $X, \Omega_{n}^{\mathrm{SO}}(X)$, are obtained from maps $f: M^{n} \rightarrow X$ of closed smooth $n$-manifolds into $X$, under the equivalence relation of bordism $([\mathbf{C F 1}])$. In this way, one builds a homology theory $\Omega_{*}^{\mathrm{SO}}(\cdot)$, for which $\Omega_{*}^{\mathrm{SO}}$ (point) is the oriented bordism ring already utilized. By Cartesian product, $\Omega_{*}^{\mathrm{SO}}(\cdot)$ is a multiplicative homology theory, and $\Omega_{*}^{\mathrm{SO}}(X)$ is a graded module over $\Omega_{*}^{\mathrm{SO}}$.

It is an advantage to invert 2 , since $\Omega_{*}^{\mathrm{SO}}\left[\frac{1}{2}\right]$ is a polynomial ring

$$
\Omega_{*}^{\mathrm{SO}}\left[\frac{1}{2}\right]=\mathbf{Z}\left[\frac{1}{2}\right]\left[x_{4}, x_{8}, x_{12}, \ldots\right] .
$$

Moreover, the forgetful homomorphism

$$
\Omega_{*}^{\text {Spin }} \rightarrow \Omega_{*}^{\text {SO }}
$$

from spin bordism to oriented bordism becomes an isomorphism when one inverts 2 ; all torsion in both bordism rings has order 2 (see $[\mathbf{S t}]$ ).

Proposition 3.2. It is possible to choose as polynomial generators $x_{4 n}$ of $\Omega_{*}^{\mathrm{SO}}\left[\frac{1}{2}\right]$ the bordism classes $x_{4}=\left[\mathbf{C P}^{2}\right], x_{8}=\left[\mathbf{H P}^{2}\right]$, and suitable $x_{4 n}=\left[M^{4 n}\right]$ $(n \geq 3)$ with $M^{4 n}$ killed by all elliptic genera.

Proof. One knows ([St, p. 180] that

$$
\Omega_{*}^{\mathrm{SO}} / \text { torsion }=\mathbf{Z}\left[x_{4}, x_{8}, \ldots\right]
$$

with $x_{4 n}=\left[M^{4 n}\right]$ a generator if and only if

$$
s_{n}(\mathcal{P})\left[M^{4 n}\right]= \begin{cases} \pm p & \text { if } 2 n=p^{s}-1, p \text { prime } \\ \pm 1 & \text { otherwise }\end{cases}
$$

Hence to obtain polynomial generators for $\Omega_{*}^{\mathrm{SO}}\left[\frac{1}{2}\right]$ we require that

$$
s_{n}(\mathcal{P})\left[M^{4 n}\right]=\left\{\begin{aligned}
\pm 2^{a} p & \text { if } 2 n=p^{s}-1, p \text { prime } \\
\pm 2^{a} & \text { otherwise. }
\end{aligned}\right.
$$


Since $s_{1}(\mathcal{P})\left[\mathbf{C P}^{2}\right]=3$ and $s_{2}(\mathcal{P})\left[\mathbf{H P}^{2}\right]=-10=-2 \cdot 5$, we can choose $x_{4}=$ $\left[\mathbf{C P}^{2}\right]$ and $x_{8}=\left[\mathbf{H P}^{2}\right]$.

It remains to find generators $\left[M^{4 n}\right], n \geq 3$, killed by all elliptic genera. Let $x_{4 n}^{\prime}(n \geq 3)$ be arbitrary generators, and notice that by Proposition 2.6 there is a polynomial

$$
Q_{n}(\delta, \varepsilon) \in \mathbf{Z}\left[\frac{1}{2}\right][\delta, \varepsilon]
$$

so that for every elliptic genus with parameters $\delta$ and $\varepsilon$

$$
\varphi\left(x_{4 n}^{\prime}\right)=Q_{n}(\delta, \varepsilon) .
$$

Take $x_{4 n}=\left[M^{4 n}\right]$ to be $x_{4 n}^{\prime}-Q_{n}\left(x_{4}, x_{8}\right)$, so that $x_{4 n}$ is also an acceptable polynomial generator for $\Omega_{*}^{\mathrm{SO}}\left[\frac{1}{2}\right]$ which is killed by all elliptic genera.

Remark 3.3. Ochanine ([O] $]$ ) has proved that every manifold $\mathbf{C P}\left(\xi^{2 m}\right)$, with $\xi^{2 m} \rightarrow B$ a smooth complex vector bundle of even rank over a closed oriented base, is killed by all elliptic genera. One can choose the polynomial generators $x_{4 n}=\left[M^{4 n}\right]$ for $n \geq 3$ in the ring $\Omega_{*}^{\mathrm{SO}}\left[\frac{1}{2}\right]$ with $M^{4 n}$ of the form $\operatorname{CP}\left(\xi^{2 m}\right)$.

3.4. It is now immediate that if $I_{*}$ denotes the ideal $\left(x_{12}, x_{16}, \ldots\right)$ in $\Omega_{*}^{\mathrm{SO}}\left[\frac{1}{2}\right]$, and $\varphi: \Omega_{*}^{\mathrm{SO}} \rightarrow \mathbf{Q}[\delta, \varepsilon]$ is a universal elliptic genus with parameters $\delta, \varepsilon$, then $\varphi$ induces an isomorphism

$$
\Omega_{*}^{\mathrm{SO}}\left[\frac{1}{2}\right] / I_{*} \stackrel{\sim}{\longrightarrow} \mathbf{Z}\left[\frac{1}{2}\right][\delta, \varepsilon] .
$$

3.5. One can produce homology theories to order, starting from a bordism theory, by a construction due to Sullivan and Baas $([\mathbf{B}])$. In our case, we begin with $\Omega_{*}^{\mathrm{SO}}(\cdot)\left[\frac{1}{2}\right]$, and the sequence (singularity set)

$$
\Sigma=\left\{x_{12}, x_{16}, \ldots\right\}
$$

where the generators are chosen as in Proposition 3.2. Noting that the sequence $x_{12}, x_{16}, \ldots$ is a regular sequence in the ring $\Omega_{*}^{\mathrm{SO}}\left[\frac{1}{2}\right]$, generating the ideal $I_{*}$, one obtains a homology theory

$$
\Omega_{*}^{\mathrm{SO}, \Sigma}\left[\frac{1}{2}\right](\cdot)
$$

with coefficient ring

$$
\Omega_{*}^{\mathrm{SO}, \Sigma}\left[\frac{1}{2}\right](\text { point })=\Omega_{*}^{\mathrm{SO}}\left[\frac{1}{2}\right] / I_{*} \stackrel{\sim}{\longrightarrow} \mathrm{M}_{*},
$$

where

$$
\mathrm{M}_{*}=\mathbf{Z}\left[\frac{1}{2}\right][\delta, \varepsilon] .
$$

Moreover, since 2 has been inverted, one obtains a multiplicative homology theory and a multiplicative natural transformation

$$
\Omega_{*}^{\mathrm{SO}}(\cdot)\left[\frac{1}{2}\right] \rightarrow \Omega_{*}^{\mathrm{SO}, \Sigma}\left[\frac{1}{2}\right](\cdot),
$$

since the obstructions to the existence of a product with these properties are 2-primary $[\mathbf{M r}]$. 
We shall write

$$
\mathrm{M}_{*}(\cdot)=\Omega_{*}^{\mathrm{SO}, \Sigma}\left[\frac{1}{2}\right](\cdot),
$$

and so have constructed a homology theory with coefficient ring $\mathrm{M}_{*}$. By localization, one can obtain further homology theories with coefficient rings (1.1), as was indicated in the introduction. However, this is such a general construction that one should seek further evidence that we have defined an important class of homology theories.

\section{Applying the exact functor theorem to localizations of $\mathbf{Z}\left[\frac{1}{2}\right][\delta, \varepsilon]$}

4.1. The natural setting for the exact functor theorem is complex bordism theory $\Omega_{*}^{\mathrm{U}}(\cdot)$, whose coefficient ring $\Omega_{*}^{\mathrm{U}}$ has already been mentioned in $\S 2$ in connection with formal groups. Let $\varphi: \Omega_{*}^{\mathrm{U}} \rightarrow R$ be a ring homomorphism to a graded commutative ring with unit, classifying a formal group $F(x, y)$ over $R$. We view $R$ as an $\Omega_{*}^{\mathrm{U}}$-module via $\varphi$, and ask if

$$
\Omega_{*}^{\mathrm{U}}(\cdot) \otimes_{\Omega_{*}^{\mathrm{U}}} R
$$

is a homology theory; when this is the case, we obtain a multiplicative homology theory with coefficient ring $R$.

To the formal group $F$ over $R$, we attach the power series

$$
[n](x)=n x+\ldots
$$

given by $[n](x)=F([n-1](x), x)$ for all $n \in \mathbf{Z}$. For each prime $p$ we write

$$
[p](x)=p x+\cdots+u_{1} x^{p}+\cdots+u_{n} x^{p^{n}}+\cdots .
$$

In case $R$ is a field of characteristic $p$, the formal group $F$ is said to have height $h$ if $u_{i}=0$ in $R$ for $i<h$ and $u_{h} \neq 0$. For the universal formal group $F_{U}$ over $\Omega_{*}^{\mathrm{U}}$, the element $u_{n}$ belongs to $\Omega_{2\left(p^{n}-1\right)}^{\mathrm{U}}$.

In order that $\Omega_{*}^{\mathrm{U}}(\cdot) \otimes_{\Omega_{*}^{\mathrm{U}}} R$ be a homology theory, it is only necessary that exactness be preserved by tensoring with $R$. There is a criterion for this to hold.

Exact Functor Theorem $4.2([\mathbf{L} 1])$. In order that $\Omega_{*}^{\mathrm{U}}(\cdot) \otimes_{\Omega_{*}^{\mathrm{U}}} R$ be a homology theory, it suffices that for each prime $p$, the sequence $p, u_{1}, \ldots, u_{n}, \ldots$ of elements in $\Omega_{*}^{\mathrm{U}}$ be a regular sequence on $R$. That is, we require that multiplication by $p$ on $R$, and by $u_{n}$ on $R /\left(p R+\cdots+u_{n-1} R\right)$ for $n \geq 1$, be injective.

REMARKS 4.3. a. We can just as well state the criterion in terms of the sequence $p, u_{1}, \ldots, u_{n}, \ldots$ of elements of $R$ assigned to the formal group over $R$.

b. The exact functor theorem also applies to modules over $\Omega_{*}^{\mathrm{U}}$, with the same statement.

c. The converse to the exact functor theorem has been proved by Rudyak $[\mathbf{R u}]$. 
d. The proof of the exact functor theorem makes use of stable operations in complex bordism theory, and the determination of the prime ideals in $\Omega_{*}^{\mathrm{U}}$ which are invariant under these operations.

4.4. It is well-known that the evident forgetful natural transformation

$$
\Omega_{*}^{\mathrm{U}}(\cdot) \rightarrow \Omega_{*}^{\mathrm{SO}}(\cdot)
$$

induces an isomorphism

$$
\Omega_{*}^{\mathrm{U}}(\cdot) \otimes_{\Omega_{*}^{\mathrm{U}}} \Omega_{*}^{\mathrm{SO}}\left[\frac{1}{2}\right] \stackrel{\sim}{\longrightarrow} \Omega_{*}^{\mathrm{SO}}(\cdot)\left[\frac{1}{2}\right] .
$$

For one proof, see [L1, p. 598]. It follows that, with

$$
\varphi: \Omega_{*}^{\mathrm{SO}} \rightarrow \mathrm{M}_{*}=\mathbf{Z}\left[\frac{1}{2}\right][\delta, \varepsilon]
$$

a universal elliptic genus, we have

$$
\Omega_{*}^{\mathrm{U}}(\cdot) \otimes_{\Omega_{*}^{\mathrm{U}}} \mathrm{M}_{*} \cong \Omega_{*}^{\mathrm{SO}}(\cdot) \otimes_{\Omega_{*}^{\mathrm{SO}}} \mathrm{M}_{*} .
$$

Thus, for the proof of Theorem 1.2, it will suffice to show that the criterion of the exact functor theorem applies to the localizations (1.1), with the formal group $F_{E}(x, y)$ of Euler understood.

4.5. We begin with $R=\mathrm{M}_{*}\left[\Delta^{-1}\right]$. We need to check the criterion for all odd primes, since it is clear for $p=2$. With $p$ an odd prime, we shall show that $p, u_{1}, u_{2}$ is a regular sequence on $R$, with $u_{2}$ a unit in

$$
R /\left(p R+u_{1} R\right) .
$$

Plainly, $p$ acts injectively on $R$, and so we next consider the action of $u_{1}$ on

$$
\mathbf{F}_{p}\left[\delta, \varepsilon, \Delta^{-1}\right] .
$$

We now refer to $[\mathbf{L 3}]$, where a thorough study has been made of the issues at stake here. Writing

$$
\left(1-2 \delta x^{2}+\varepsilon x^{4}\right)^{-\frac{1}{2}}=\sum_{n \geq 0} P_{n}(\delta, \varepsilon) x^{2 n},
$$

we have

$$
u_{1} \equiv P_{(p-1) / 2}(\delta, \varepsilon) \quad \bmod p
$$

$([\mathbf{L 3},(1.2)])$. Note that $P_{n}(1,1)=1$ for all $n$, so certainly $u_{1} \not \equiv 0 \bmod p$.

We next argue that $u_{2}$ is a unit in

$$
\mathbf{Z}\left[\delta, \varepsilon, \Delta^{-1}\right] /\left(p, u_{1}\right) .
$$

If $u_{2}$ were not a unit in this ring, it would lie in a maximal ideal. Hence we would obtain a field $K$ of characteristic $p$ and an elliptic curve in Jacobi quartic form

$$
y^{2}=1-2 \delta x^{2}+\varepsilon x^{4}
$$


over $K$, for which the corresponding formal group law has $u_{1}=u_{2}=0$. But it is well-known ([Si, Cor. IV.7.5]) that the height of the formal group of an elliptic curve over a field of characteristic $p>0$ is 1 or 2 ; i.e., if $u_{1}=0$ then $u_{2} \neq 0$. This contradiction completes the proof in this case.

4.6. More explicitly, we have congruences modulo $\left(p, u_{1}\right)$ :

$$
\begin{aligned}
u_{2} & \equiv(-1)^{(p-1) / 2} \varepsilon^{\left(p^{2}-1\right) / 4}, \\
\left(\delta^{2}-\varepsilon\right)^{\left(p^{2}-1\right) / 4} & \equiv \varepsilon^{\left(p^{2}-1\right) / 4} ;
\end{aligned}
$$

see $[\mathbf{L 3}$, Thm. 1] and the following discussion. Recalling that

$$
\Delta=\varepsilon\left(\delta^{2}-\varepsilon\right)^{2},
$$

we see that $u_{2}$ is a unit in

$$
\mathbf{Z}\left[\delta, \varepsilon, \Delta^{-1}\right] /\left(p, u_{1}\right),
$$

as required.

Moreover, we see that modulo $\left(p, u_{1}\right)$, inverting $\delta^{2}-\varepsilon$ is equivalent to inverting $\varepsilon$, and so also to inverting the discriminant $\Delta$. Thus in both $\mathrm{M}_{*}\left[\varepsilon^{-1}\right]$ and $\mathrm{M}_{*}\left[\left(\delta^{2}-\varepsilon\right)^{-1}\right]$, we see that $p, u_{1}, u_{2}$ is a regular sequence with $u_{2}$ a unit modulo $\left(p, u_{1}\right)$. Hence the exact functor theorem applies to these localizations of $\mathrm{M}_{*}$ as well, completing the proof of Theorem 1.2.

4.7. In the previous section, we used bordism with singularities to construct a homology theory $\mathrm{M}_{*}(\cdot)$ and a natural transformation

$$
\Phi: \Omega_{*}^{\mathrm{SO}}(\cdot) \rightarrow \mathrm{M}_{*}(\cdot)
$$

extending the universal elliptic genus

$$
\varphi: \Omega_{*}^{\mathrm{SO}} \rightarrow \mathrm{M}_{*} .
$$

In view of Theorem 1.2, this leads to a natural transformation

$$
\Omega_{*}^{\mathrm{SO}}(\cdot) \otimes_{\Omega_{*}^{\mathrm{SO}}} \mathrm{M}_{*}\left[\Delta^{-1}\right] \rightarrow \mathrm{M}_{*}(\cdot)\left[\Delta^{-1}\right]
$$

between homology theories, which is an isomorphism on coefficient rings. By the comparison theorem for homology theories (easily proved by induction on the number of cells for finite complexes, and then by taking direct limits for arbitrary $\mathrm{CW}$-complexes) we obtain an isomorphism of homology theories. Evidently, we obtain the same conclusion by inverting merely $\varepsilon$ or $\delta^{2}-\varepsilon$. Thus we have established Conner-Floyd theorems for all the periodic elliptic homology theories obtained by localization from $\mathrm{M}_{*}(\cdot)$. 
4.8. We turn to relations to KO-theory with 2 inverted. We choose to deal with KO-homology, which has period 4 when one inverts 2 . The coefficient ring is

with $\gamma$ of degree 4 .

$$
\mathrm{KO}_{*}\left[\frac{1}{2}\right]=\mathbf{Z}\left[\frac{1}{2}\right]\left[\gamma, \gamma^{-1}\right]
$$

Proposition 4.9. There are natural transformations

$$
\begin{aligned}
& \nu_{s}: \Omega_{*}^{\mathrm{SO}}(\cdot) \otimes_{\Omega_{*}^{\mathrm{SO}}} \mathrm{M}_{*}\left[\varepsilon^{-1}\right] \rightarrow \mathrm{KO}_{*}(\cdot)\left[\frac{1}{2}\right] \\
& \nu_{d}: \Omega_{*}^{\mathrm{SO}}(\cdot) \otimes_{\Omega_{*}^{\mathrm{SO}}} \mathrm{M}_{*}\left[\left(\delta^{2}-\varepsilon\right)^{-1}\right] \rightarrow \mathrm{KO}_{*}(\cdot)\left[\frac{1}{2}\right]
\end{aligned}
$$

so that on coefficient rings the compositions

$$
\begin{aligned}
\Omega_{*}^{\mathrm{SO}} \stackrel{\varphi}{\longrightarrow} \mathrm{M}_{*}\left[\varepsilon^{-1}\right] \stackrel{\nu_{s}}{\longrightarrow} \mathrm{KO}_{*}\left[\frac{1}{2}\right] & =\mathbf{Z}\left[\frac{1}{2}\right]\left[\gamma, \gamma^{-1}\right] \\
\Omega_{*}^{\mathrm{SO}} \stackrel{\varphi}{\longrightarrow} \mathrm{M}_{*}\left[\left(\delta^{2}-\varepsilon\right)^{-1}\right] \stackrel{\nu_{d}}{\longrightarrow} \mathrm{KO}_{*}\left[\frac{1}{2}\right] & =\mathbf{Z}\left[\frac{1}{2}\right]\left[\gamma, \gamma^{-1}\right]
\end{aligned}
$$

send a bordism class $\left[M^{4 n}\right]$ to $\operatorname{sign}\left(M^{4 n}\right) \gamma^{n}$ and to $\hat{\mathrm{A}}\left(M^{4 n}\right) \gamma^{n}$, respectively.

PRoOF. There are well-known natural transformations

$$
\begin{aligned}
& \mu_{d}: \Omega_{*}^{\mathrm{Spin}}(\cdot) \rightarrow \mathrm{KO}_{*}(\cdot) \\
& \mu_{s}: \Omega_{*}^{\mathrm{SO}}(\cdot) \rightarrow \mathrm{KO}_{*}(\cdot)\left[\frac{1}{2}\right]
\end{aligned}
$$

due to Atiyah and Milnor [Mi] and to Sullivan $[\mathbf{S u}]$, which on coefficient rings send a bordism class $\left[M^{4 n}\right]$ to $\hat{\mathrm{A}}\left(M^{4 n}\right) \gamma^{n}$ and $\operatorname{sign}\left(M^{4 n}\right) \gamma^{n}$, respectively. Further, $\mu_{d}$ and $\mu_{s}$ induce natural equivalences

$$
\begin{array}{r}
\tilde{\mu}_{d}: \Omega_{*}^{\mathrm{Spin}}(\cdot) \otimes_{\Omega_{*}^{\mathrm{Spin}}} \mathrm{KO}_{*}\left[\frac{1}{2}\right] \stackrel{\sim}{\longrightarrow} \mathrm{KO}_{*}(\cdot)\left[\frac{1}{2}\right], \\
\tilde{\mu}_{s}: \Omega_{*}^{\mathrm{SO}}(\cdot) \otimes_{\Omega_{*}^{\text {SO }}} \mathrm{KO}_{*}\left[\frac{1}{2}\right] \stackrel{\sim}{\longrightarrow} \mathrm{KO}_{*}(\cdot)\left[\frac{1}{2}\right] ;
\end{array}
$$

see $[$ CF2 $]$ for the first of these (they really used symplectic bordism, but $\Omega_{*}^{\mathrm{Sp}} \rightarrow$ $\Omega_{*}^{\text {Spin }}$ becomes an isomorphism when 2 is inverted), and [L1, Ex. 3.4] for the second.

Next, define ring homomorphisms

$$
\begin{aligned}
& \nu_{s}: \mathrm{M}_{*}\left[\varepsilon^{-1}\right] \rightarrow \mathbf{Z}\left[\frac{1}{2}\right]\left[\gamma, \gamma^{-1}\right], \quad \delta \mapsto \gamma, \quad \varepsilon \mapsto \gamma^{2} ; \\
& \nu_{d}: \mathrm{M}_{*}\left[\left(\delta^{2}-\varepsilon\right)^{-1}\right] \rightarrow \mathbf{Z}\left[\frac{1}{2}\right]\left[\gamma, \gamma^{-1}\right], \quad \delta \mapsto-\frac{1}{8} \gamma, \quad \varepsilon \mapsto 0 .
\end{aligned}
$$

These extend to

$$
\begin{aligned}
\nu_{s}: \Omega_{*}^{\mathrm{SO}}(\cdot) \otimes_{\Omega_{*}^{\mathrm{SO}}} \mathrm{M}_{*}\left[\varepsilon^{-1}\right] & \rightarrow \Omega_{*}^{\mathrm{SO}}(\cdot) \otimes_{\Omega_{*}^{\mathrm{SO}}} \mathrm{KO}_{*}\left[\frac{1}{2}\right], \\
\nu_{d}: \Omega_{*}^{\mathrm{SO}}(\cdot) \otimes_{\Omega_{*}^{\mathrm{SO}}} \mathrm{M}_{*}\left[\left(\delta^{2}-\varepsilon\right)^{-1}\right] & \rightarrow \Omega_{*}^{\mathrm{SO}}(\cdot) \otimes_{\Omega_{*}^{\mathrm{SO}}} \mathrm{KO}_{*}\left[\frac{1}{2}\right] .
\end{aligned}
$$

Viewing $\tilde{\mu}_{s}$ and $\tilde{\mu}_{d}$ as identifications, we obtain the desired natural transformations from elliptic homology to KO-theory with 2 inverted.

The composition (4.10) is an elliptic genus sending $\mathbf{C} \mathbf{P}^{2}$ to $\gamma$ and $\mathbf{H} \mathbf{P}^{2}$ to $\gamma^{2}$, hence sends $M^{4 n}$ to $\operatorname{sign}\left(M^{4 n}\right) \gamma^{n}$. Similarly, the composition (4.11) is an elliptic 
genus killing $\mathbf{H P}^{2}$ and sending $\mathbf{C P}^{2}$ to $-\frac{1}{8} \gamma=\hat{\mathrm{A}}(\mathbf{C P}) \gamma$, thus sending $M^{4 n}$ to $\hat{\mathrm{A}}\left(M^{4 n}\right) \gamma^{n}$.

4.12. We can obtain further periodic homology theories, corresponding to the Weierstrass equations (1.3) and (1.4)

$$
\begin{aligned}
y^{2} & =4 x^{3}-g_{2} x-g_{3} \\
y^{2}+a_{1} x y+a_{3} y & =x^{3}+a_{2} x^{2}+a_{1} x+a_{6},
\end{aligned}
$$

the homogeneous Legendre normal form

$$
y^{2}=x(x-\lambda)(x-\mu)
$$

and the homogeneous Deuring normal form

$$
y^{2}+\alpha x y+\beta y=x^{3}
$$

([Si, p. 327]). In these cases, the coefficient rings are

$$
\begin{gathered}
\mathbf{Z}\left[\frac{1}{6}\right]\left[g_{2}, g_{3}, \Delta^{-1}\right], \\
\mathbf{Z}\left[a_{1}, a_{2}, a_{3}, a_{4}, a_{6}, \Delta^{-1}\right], \\
\mathbf{Z}\left[\frac{1}{2}\right]\left[\lambda, \mu, \Delta^{-1}\right], \\
\mathbf{Z}\left[\frac{1}{3}\right]\left[\alpha, \beta, \Delta^{-1}\right] .
\end{gathered}
$$

(For the Legendre form, wt $\lambda=$ wt $\mu=2$ and $\Delta=16 \lambda^{2} \mu^{2}(\lambda-\mu)^{2}$; for the Deuring form, wt $\alpha=1$, wt $\beta=3$ and $\Delta=\beta^{3}\left(\alpha^{3}-27 \beta\right)$.) In all cases, we apply the exact functor theorem, using the formal group as given in $[\mathbf{S i}, \mathbf{C h}$. IV] to make the desired coefficient ring an algebra over $\Omega_{*}^{\mathrm{U}}$.

For example, consider the classical Weierstrass form (1.3). We must show that the exact functor theorem applies to $\mathbf{Z}\left[\frac{1}{6}\right]\left[g_{2}, g_{3}, \Delta^{-1}\right]$, and so need only consider primes $p>3$. Plainly $p$ is not a zero-divisor, so we next consider $u_{1}$ (the Hasse invariant) in $\mathbf{F}_{p}\left[g_{2}, g_{3}, \Delta^{-1}\right]$. It is well-known that $u_{1} \not \equiv 0 \bmod p$, since otherwise all elliptic curves in characteristic p would be supersingular! Next, we can argue that $u_{2}$ is a unit in

$$
\mathbf{F}_{p}\left[g_{2}, g_{3}, \Delta^{-1}\right]
$$

exactly as at the end of $\S 4.5$ above; indeed, a more precise result, that

$$
u_{2} \equiv(-1)^{(p-1) / 2} \Delta^{\left(p^{2}-1\right) / 12} \bmod \left(p, u_{1}\right),
$$

has been obtained in $[\mathbf{L 3}]$.

The argument is the same for the other normal forms. Note that we need not invert any primes if we use the general Weierstrass equation (1.4), at the price of having to permit all five coefficients to appear as indeterminates. 


\section{Modular forms for $\Gamma_{0}(2)$}

We begin with some preliminaries on modular forms and modular functions, referring to $[\mathbf{K}, \mathrm{Ch}$. III $]$ for background and further information.

5.1. Write $\Gamma=\mathrm{SL}_{2}(\mathbf{Z})$ for the modular group, and put

$$
\Gamma_{0}(2)=\left\{\left(\begin{array}{ll}
a & b \\
c & d
\end{array}\right) \in \Gamma: c \equiv 0 \quad \bmod 2\right\} \text {. }
$$

$\Gamma_{0}(2)$ is a subgroup of $\Gamma$ of index 3 , not normal and having as conjugates

$$
\Gamma^{0}(2)=\left\{\left(\begin{array}{ll}
a & b \\
c & d
\end{array}\right) \in \Gamma: b \equiv 0 \quad \bmod 2\right\}
$$

and the "theta group"

$$
\Gamma_{\theta}=\text { all }\left(\begin{array}{ll}
a & b \\
c & d
\end{array}\right) \in \Gamma \text { congruent to } \pm\left(\begin{array}{ll}
1 & 0 \\
0 & 1
\end{array}\right) \text { or to } \pm\left(\begin{array}{ll}
0 & 1 \\
1 & 0
\end{array}\right) \quad \bmod 2 .
$$

$\Gamma_{0}(2)$ acts on the upper half-plane $\mathcal{H}$ by

$$
\left(\begin{array}{ll}
a & b \\
c & d
\end{array}\right) \tau=\frac{a \tau+b}{c \tau+d}
$$

a fundamental domain has been given in the introduction, namely

$$
\left\{z: \operatorname{Im} z>0,0<\operatorname{Re} z<1,\left|z-\frac{1}{2}\right|>\frac{1}{2}\right\} .
$$

Note that $\left(\begin{array}{ll}1 & 1 \\ 0 & 1\end{array}\right) \in \Gamma_{0}(2)$, corresponding to $\tau \mapsto \tau+1$. $\Gamma_{0}(2)$ has the two cusps 0 and $\infty$.

5.2. By a modular function of integer weight $k$ for $\Gamma_{0}(2)$, one means a meromorphic function $f: \mathcal{H} \rightarrow \mathbf{C}$ satisfying

$$
f\left(\frac{a \tau+b}{c \tau+d}\right)=(c \tau+d)^{k} f(\tau)
$$

for $\left(\begin{array}{ll}a & b \\ c & d\end{array}\right) \in \Gamma_{0}(2)$ and $\tau \in \mathcal{H}$, as well as the following conditions at the cusps. Since $\left(\begin{array}{ll}1 & 1 \\ 0 & 1\end{array}\right) \in \Gamma_{0}(2)$ we have $f(\tau+1)=f(\tau)$, so can write

$$
f(\tau)=\sum_{-\infty}^{\infty} a_{n} q^{n} \quad\left(q=e^{2 \pi i \tau}\right)
$$

we require that for some $n_{0}, a_{n}=0$ if $n<n_{0}$, i.e. $f$ is meromorphic at $\infty$. Further, $g(\tau):=\tau^{-k} f(-1 / \tau)$ satisfies $g(\tau+2)=g(\tau)$ so we can write

$$
\tau^{-k} f(-1 / \tau)=\sum_{-\infty}^{\infty} b_{n} q_{2}^{n} \quad\left(q_{2}=e^{\pi i \tau}\right)
$$


we require that for some $n_{0}, b_{n}=0$ if $n<n_{0}$, i.e. $f$ is meromorphic at the cusp $\tau=0$. One refers to these series as the q-expansions of $f$ at the cusps $\infty$ and 0 . It is customary to identify $f$ with its $q$-expansion at $\infty$.

We call $f$ a modular form of integral weight $k \geq 0$ for $\Gamma_{0}(2)$ if $f$ is holomorphic on $\mathcal{H}$ and at the cusps:

$$
\begin{aligned}
f(\tau) & =\sum_{n \geq 0} a_{n} q^{n}, \\
\tau^{-k} f(-1 / \tau) & =\sum_{n \geq 0} b_{n} q_{2}{ }^{n} .
\end{aligned}
$$

We write $\mathrm{M}_{k}\left(\Gamma_{0}(2)\right)$ for the vector space of modular forms for $\Gamma_{0}(2)$ of weight $k$; it is zero for $k$ odd, since $-\left(\begin{array}{ll}a & b \\ c & d\end{array}\right)$ belongs to $\Gamma_{0}(2)$ when $\left(\begin{array}{ll}a & b \\ c & d\end{array}\right)$ does. The modular forms give rise to a graded ring $\mathrm{M}_{*}\left(\Gamma_{0}(2)\right)$.

5.3. The work of a number of authors has led to a consensus that the fundamental elliptic genus takes its values in $\mathrm{M}_{*}\left(\Gamma_{0}(2)\right)$; see $[\mathbf{L S}, \mathbf{C C}, \mathbf{Z}]$. That is, one can construct an elliptic genus

$$
\varphi: \Omega_{*}^{\mathrm{SO}} \rightarrow \mathbf{Q}[[q]]
$$

whose values on any bordism class $\left[M^{4 k}\right]$ is a modular form for $\Gamma_{0}(2)$ of weight $2 k$, with constant term $\hat{\mathrm{A}}\left(M^{4 k}\right)$. In fact, the $q$-expansion coefficients all lie in $\mathbf{Z}\left[\frac{1}{2}\right]$, and are integral when $M^{4 k}$ is a spin manifold. One has precise additive formulas for the parameters $\delta$ and $\varepsilon([\mathbf{Z}])$ :

$$
\begin{aligned}
& \delta=-\frac{1}{8}-3 \sum_{n \geq 1}\left(\sum_{\substack{d \mid n \\
d \text { odd }}} d\right) q^{n}, \\
& \varepsilon=\sum_{n \geq 1}\left(\sum_{\substack{d \mid n \\
\frac{n}{d} \text { odd }}} d^{3}\right) q^{n}
\end{aligned}
$$

these are modular forms of weights 2 and 4 , respectively, which are algebraically independent and satisfy

$$
\mathrm{M}_{*}\left(\Gamma_{0}(2)\right)=\mathbf{C}[\delta, \varepsilon] .
$$

5.4. We shall now express $\delta$ and $\varepsilon$ in terms of theta constants, and so obtain infinite product expansions for $\varepsilon, \delta^{2}-\varepsilon$ and the discriminant $\Delta=\varepsilon\left(\delta^{2}-\varepsilon\right)^{2}$. This discussion relies heavily on Chapter $\mathrm{V}$ in $[\mathbf{C n}]$, dealing with theta functions. For a related treatment, see $[\mathbf{L} 2, \S 4]$ and $[\mathbf{L 3}, \S 5]$.

Viewing $\tau$ as a parameter in the upper half-plane, Zagier $([\mathbf{Z}])$ shows that $s(u)=s(u ; \tau):=g^{-1}(u)$ (recall that $g^{-1}(u)$ is the inverse under composition of the logarithm $u=g(x)$ of the elliptic genus) is an odd elliptic function of order 2 with period lattice

$$
4 \pi i(\mathbf{Z}+\mathbf{Z} \tau)
$$


satisfying $s^{\prime}(0)=1$ and

$$
s^{\prime}(u)^{2}=1-2 \delta(\tau) s(u)^{2}+\varepsilon(\tau) s(u)^{4} .
$$

$s(u)$ has simple zeros at $u=0$ and $u=2 \pi i$, and simple poles at $2 \pi i \tau$ and $2 \pi i(1+\tau)$.

Now let $\wp$ be the Weierstrass function for the same lattice, and put

$$
\omega_{1}=4 \pi i, \omega_{2}=4 \pi i \tau,
$$

so that $\tau=\omega_{2} / \omega_{1}$ and the lattice is generated by $\omega_{1}$ and $\omega_{2}$. Then $\wp$ has the half-period values

$$
\begin{aligned}
& e_{1}=\wp\left(\omega_{1} / 2\right)=\wp(2 \pi i) \\
& e_{2}=\wp\left(\omega_{2} / 2\right)=\wp(2 \pi i \tau) \\
& e_{3}=\wp\left(\omega_{3} / 2\right)=\wp(2 \pi i(1+\tau)),
\end{aligned}
$$

where $\omega_{3}=\omega_{1}+\omega_{2}$ and $e_{1}+e_{2}+e_{3}=0$. One sees easily, by comparing divisors, that

$$
s(u)=-2 \frac{\wp(u)-e_{1}}{\wp^{\prime}(u)} .
$$

LeMma 5.5. One has

$$
\begin{aligned}
\delta & =3 e_{1} \\
\varepsilon & =\left(e_{2}-e_{3}\right)^{2} \\
\delta^{2}-\varepsilon & =4\left(e_{1}-e_{2}\right)\left(e_{1}-e_{3}\right) \\
\Delta & =16\left(e_{1}-e_{2}\right)^{2}\left(e_{1}-e_{3}\right)^{2}\left(e_{2}-e_{3}\right)^{2},
\end{aligned}
$$

where $\Delta=\varepsilon\left(\delta^{2}-\varepsilon\right)^{2}$.

This is proved by a straightforward comparison of coefficients of $s(u)$ and $\wp(u)$, and is left to the reader (see $[\mathbf{L 3}, \S 5]$ ). The expression for the discriminant is classical.

We now follow Chandrasekharan $[\mathbf{C n}]$, still taking $q=e^{2 \pi i \tau}$ rather than $q=e^{\pi i \tau}$ as in $[\mathbf{C n}, \mathrm{Ch} . \mathrm{V}]$. The theta constants $\theta_{1}(\tau), \theta_{2}(\tau)$ and $\theta_{3}(\tau)$ have the $q$-expansions

$$
\begin{aligned}
& \theta_{1}=2 q^{1 / 8} \sum_{n \geq 0} q^{n(n+1) / 2} \\
& \theta_{2}=1+2 \sum_{n \geq 1}(-1)^{n} q^{n^{2} / 2} \\
& \theta_{3}=1+2 \sum_{n \geq 1} q^{n^{2} / 2}
\end{aligned}
$$


and the infinite product representations (for $|q|<1$ )

$$
\begin{aligned}
& \theta_{1}=2 q^{1 / 8} \prod_{n \geq 1}\left(1-q^{n}\right)\left(1+q^{n}\right)^{2} \\
& \theta_{2}=\prod_{n \geq 1}\left(1-q^{n}\right)\left(1-q^{(2 n-1) / 2}\right)^{2} \\
& \theta_{3}=\prod_{n \geq 1}\left(1-q^{n}\right)\left(1+q^{(2 n-1) / 2}\right)^{2} .
\end{aligned}
$$

In terms of the $\theta_{i}$ 's we have $[\mathbf{C n}$, p. 69])

$$
\begin{aligned}
e_{2}-e_{1} & =\frac{1}{16} \theta_{3}^{4}(\tau) \\
e_{3}-e_{1} & =\frac{1}{16} \theta_{2}^{4}(\tau) \\
e_{2}-e_{3} & =\frac{1}{16} \theta_{1}^{4}(\tau),
\end{aligned}
$$

from which one can observe the well-known relation

$$
\theta_{1}^{4}+\theta_{2}^{4}=\theta_{3}^{4}
$$

It follows at once that

$$
e_{1}=-\frac{1}{48}\left(\theta_{2}^{4}+\theta_{3}^{4}\right)
$$

In view of the lemma, we conclude that

Proposition 5.6. One has

$$
\begin{aligned}
\delta & =-\frac{1}{16}\left(\theta_{2}{ }^{4}+\theta_{3}{ }^{4}\right), \\
\varepsilon & =\frac{1}{256} \theta_{1}^{8}, \\
\delta^{2}-\varepsilon & =\frac{1}{64} \theta_{2}^{4} \theta_{3}{ }^{4} .
\end{aligned}
$$

We further obtain the infinite product representations:

Proposition 5.7. One has

$$
\begin{aligned}
\varepsilon & =q \prod_{n=1}^{\infty}\left\{\left(1-q^{n}\right)\left(1+q^{n}\right)^{2}\right\}^{8}, \\
\delta^{2}-\varepsilon & =\frac{1}{64} \prod_{n=1}^{\infty}\left\{\left(1-q^{n}\right)\left(1-q^{2 n-1}\right)\right\}^{8}, \\
\Delta & =2^{-12} q \prod_{n=1}^{\infty}\left(1-q^{n}\right)^{24} .
\end{aligned}
$$


Since $q=e^{2 \pi i \tau}$, we see that $\Delta=\varepsilon\left(\delta^{2}-\varepsilon\right)^{2}$ is nonzero for all $\tau \in \mathcal{H}$. The behavior at the cusp $\tau=\infty$ (i.e., $q=0$ ) is clear; the elliptic genus degenerates to the $\hat{A}$-genus.

The behavior at the other cusp, $\tau=0$, follows from the formulas ([Cn, p. 75])

$$
\begin{aligned}
& \theta_{1}(\tau)=\sqrt{i / \tau} \theta_{2}(-1 / \tau) \\
& \theta_{2}(\tau)=\sqrt{i / \tau} \theta_{1}(-1 / \tau) \\
& \theta_{3}(\tau)=\sqrt{i / \tau} \theta_{3}(-1 / \tau) .
\end{aligned}
$$

In view of Proposition 5.6, we find that

$$
\begin{aligned}
\tau^{-2} \delta(-1 / \tau) & =\frac{1}{16}\left(\theta_{1}(\tau)^{4}+\theta_{3}(\tau)^{4}\right), \\
\tau^{-4} \varepsilon(-1 / \tau) & =\frac{1}{256} \theta_{2}(\tau)^{8}, \\
\tau^{-4}\left(\delta^{2}-\varepsilon\right)(-1 / \tau) & =\frac{1}{64} \theta_{1}(\tau)^{4} \theta_{3}(\tau)^{4} ;
\end{aligned}
$$

these have constant terms $1 / 16,1 / 256$ and 0 respectively. Hence the elliptic genus degenerates (essentially) to the L-genus at this cusp.

5.8. We finally turn to the proof of Theorem 1.5, which was stated in the introduction. We begin by identifying $\mathrm{M}_{*}=\mathbf{Z}\left[\frac{1}{2}\right][\delta, \varepsilon]$ as a subring of $\mathrm{M}_{*}\left(\Gamma_{0}(2)\right)$. For any ring $R$ with $\mathbf{Z} \subset R \subset \mathbf{Q}$ we denote by

$$
\mathrm{M}_{*}^{R}\left(\Gamma_{0}(2)\right)
$$

the set of all $f \in \mathrm{M}_{*}\left(\Gamma_{0}(2)\right)$ whose homogeneous components have $q$-expansion coefficients in $R$. Evidently, $8 \delta$ and $\varepsilon$ lie in $\mathrm{M}_{*}^{\mathbf{Z}}\left(\Gamma_{0}(2)\right)$, in view of the formulas in $\S 5.3$. Hence each modular form in $\mathrm{M}_{*}$ lies in $\mathrm{M}_{*}^{\mathrm{Z}\left[\frac{1}{2}\right]}\left(\Gamma_{0}(2)\right)$. That these rings coincide follows from the

Proposition 5.9. For any ring $R$ with $\mathbf{Z} \subset R \subset \mathbf{Q}$ we have

$$
\mathrm{M}_{*}^{R}\left(\Gamma_{0}(2)\right)=R[8 \delta, \varepsilon] .
$$

Proof. In one direction, this is clear from what has been noted. Conversely, let $f \in \mathrm{M}_{2 k}^{R}\left(\Gamma_{0}(2)\right)$ and write

$$
f=\sum_{l=0}^{[k / 2]} a_{l}(-8 \delta)^{k-2 l} \varepsilon^{l},
$$

in which the individual terms have $q$-expansions beginning with $a_{l} q^{l}$. By induction on $l$, it is immediate that each $a_{l}$ lies in $R$.

We next identify $\mathrm{M}_{*}\left[\Delta^{-1}\right]$ as a ring of modular functions for $\Gamma_{0}(2)$. Note first that $\varepsilon$ has a simple zero at $\tau=\infty$, and is otherwise nonzero on $\mathcal{H} \cup\{0, \infty\}$. Similarly, $\delta^{2}-\varepsilon$ has a simple zero at $\tau=0$, and is otherwise nonzero on $\mathcal{H} \cup\{0, \infty\}$. Thus, the only zeros of $\Delta$ occur at the cusps. 
Hence, each element of $\mathrm{M}_{*}\left[\Delta^{-1}\right]$ is a modular function with $q$-expansion coefficients in $\mathbf{Z}\left[\frac{1}{2}\right]$, and is holomorphic on $\mathcal{H}$ (its only possible poles being at the cusps).

Conversely, if $f$ is a modular function which is holomorphic on $\mathcal{H}$, then $f \Delta^{N}$ is a modular form for some integer $N \geq 0$. And if all $q$-expansion coefficients of $f$ lie in $\mathbf{Z}\left[\frac{1}{2}\right]$, the same is true of $f \Delta^{N}$, so that $f \Delta^{N}$ lies in $\mathrm{M}_{*}$ and $f \in \mathrm{M}_{*}\left[\Delta^{-1}\right]$.

Similarly, each element of $\mathrm{M}_{*}\left[\varepsilon^{-1}\right]$ is a modular function with $q$-expansion coefficients in $\mathbf{Z}\left[\frac{1}{2}\right]$, which is holomorphic on $\mathcal{H} \cup\{0\}$ (it may have a pole at $\infty$ ). Conversely, if $f$ is such a modular function then $f \varepsilon^{N}$ is a modular form and so lies in $\mathrm{M}_{*}$, for some $n \geq 0$, whence $f \in \mathrm{M}_{*}\left[\varepsilon^{-1}\right]$.

Finally, in the same way one sees that $\mathrm{M}_{*}\left[\left(\delta^{2}-\varepsilon\right)^{-1}\right]$ consists of all modular functions with $q$-expansion coefficients in $\mathbf{Z}\left[\frac{1}{2}\right]$, which are holomorphic on $\mathcal{H} \cup\{\infty\}$.

REMARK 5.10. The modular functions of weight zero in $\mathrm{M}_{*}\left[\Delta^{-1}\right], \mathrm{M}_{*}\left[\varepsilon^{-1}\right]$ and $\mathrm{M}_{*}\left[\left(\delta^{2}-\varepsilon\right)^{-1}\right]$ are easily characterized as: Laurent polynomials in $\left(\delta^{2}-\varepsilon\right) / \varepsilon$, polynomials in $\left(\delta^{2}-\varepsilon\right) / \varepsilon$, and polynomials in $\varepsilon /\left(\delta^{2}-\varepsilon\right)$, respectively, the coefficients of these polynomials being in $\mathbf{Z}\left[\frac{1}{2}\right]$.

\section{REFERENCES}

[B] N. A. Baas, On bordism theory of manifolds with singularities, Math. Scand. 33 (1973), 279-302.

[Cn] K. Chandrasekharan, Elliptic Functions, Springer-Verlag, 1985.

[CC] D. V. Chudnovsky and G. V. Chudnovsky, Elliptic modular functions and elliptic genera, Topology 27 (1988), 163-170.

[CCLOS] D. V. Chudnovsky, G. V. Chudnovsky, P. S. Landweber, S. Ochanine and R. E. Stong, Integrality and divisiblity of elliptic genera (to appear).

[CF1] P. E. Conner and E. E. Floyd, Differentiable Periodic Maps, Springer-Verlag, 1964.

[CF2] - The Relation of Cobordism to K-theories, Lecture Notes in Math., vol. 28, Springer-Verlag, 1966.

[E] L. Euler, De integrationis aequationis differentialis $m d x / \sqrt{1-x^{4}}=n d y / \sqrt{1-y^{4}}$, Opera omnia XX (1), Teubner-Füssli, 1911-1976, pp. 58-79.

[F] J. Franke, On the construction of elliptic cohomology, Math. Nachr. 158 (1992), $43-65$.

[H] F. Hirzebruch, Topological Methods in Algebraic Geometry, Springer-Verlag, 1966.

[Ho] M. Hovey, Spin bordism and elliptic homology, Math. Z. (to appear).

[K] N. Koblitz, Introduction to Elliptic Curves and Modular Forms, Graduate Texts in Math, vol. 97, Springer-Verlag, 1984, 1993 (2nd ed.).

[KS] M. Kreck and S. Stolz, $\mathrm{HP}^{2}$-bundles and elliptic homology, Acta Mathematica 171 (1993), 231-261.

[L1] P. S. Landweber, Homological properties of comodules over $\mathrm{MU}_{*} \mathrm{MU}$ and $\mathrm{BP} * \mathrm{BP}$, Amer. J. Math. 98 (1976), 591-610.

[L2] Elliptic cohomology and modular forms, Elliptic Curves and Modular Forms in Algebraic Topology (Proceedings, Princeton 1986). Lecture Notes in Math., vol. 1326, Springer-Verlag, 1988, pp. 55-68.

[L3] _ Supersingular elliptic curves and congruences for Legendre polynomials, Elliptic Curves and Modular Forms in Algebraic Topology. Lecture Notes in Math., vol. 1326, Springer-Verlag, 1988, pp. 69-93. 
[LS] P. S. Landweber and R. E. Stong, Circle actions on Spin manifolds and characteristic numbers, Topology 27 (1988), 145-161.

[Ml] J. W. Milnor, Remarks concerning spin manifolds, Differential and Combinatorial Topology (S. Cairns, ed.), Princeton Univ. Press, 1965, pp. 55-62.

[MS] J. W. Milnor and J. D. Stasheff, Characteristic Classes, Annals of Math. Studies, vol. 76, Princeton Univ. Press, 1974.

[Mr] O. K. Mironov, Multiplications in cobordism theories with singularities, and Steenrodtom Dieck operations, Izv. Akad. Nauk SSSR, Ser. Mat. 42 (1978), 789-806; English transl. in Math. USSR Izvestiya 13 (1979), 89-106.

[O] S. Ochanine, Sur les genres multiplicatifs définis par des intégrales elliptiques, Topology 26 (1987), 143-151.

[O1] Elliptic genera, modular forms over $\mathrm{KO}_{*}$, and the Brown-Kervaire invariant, Math. Z. 206 (1991), 277-291.

[Q] D. Quillen, On the formal group laws of unoriented and complex cobordism theory, Bull. Amer. Math. Soc 75 (1969), 1293-1298.

[R] D. C. Ravenel, Complex Cobordism and Stable Homotopy Groups of Spheres, Academic Press, 1986.

[Ru] Yu. B. Rudyak, Exactness theorems for the cohomology theories MU, BP and $\mathrm{P}(n)$, Mat. Zametki 40 (1986), 115-126; English transl. in Math. Notes 40 (1986), 562569.

[Se] J.-P. Serre, A Course in Arithmetic, Graduate Texts in Math., vol. 7, SpringerVerlag, 1973.

[Si] J. H. Silverman, The Arithmetic of Elliptic Curves, Graduate Texts in Math., vol. 106, Springer-Verlag, 1986.

[St] R. E. Stong, Notes on Cobordism Theory, Princeton Univ. Press, 1968.

[Su] D. Sullivan, Triangulating and smoothing homotopy equivalences and homeomorphisms, Geometric Topology Seminar notes, Princeton Univ., 1967.

[Ta] C. H. Taubes, $S^{1}$ actions and elliptic genera, Comm. Math. Phys. 122 (1989), 455526.

[Th] R. Thom, Quelques propriétés globales des variétés différentiables, Comm. Math. Helv. 28 (1954), 17-86.

[W1] E. Witten, Elliptic genera and quantum field theory, Comm. Math. Phys. 109 (1987), $525-536$.

[W2] The index of the Dirac operator on loop space, Elliptic Curves and Modular Forms in Algebraic Topology, Lecture Notes in Math., vol. 1326, Springer-Verlag, 1988, pp. 161-181.

[Z] D. Zagier, Note on the Landweber-Stong elliptic genus, Elliptic Curves and Modular Forms in Algebraic Topology, Lecture Notes in Math., vol. 1326, Springer-Verlag, 1988, pp. 216-224.

Department of Mathematics, Rutgers University, New Brunswick, NJ 08903

E-mail address: landwebe@math.rutgers.edu

Department of Mathematics, University of Rochester, Rochester, Ny 14627

E-mail address: drav@troi.cc.rochester.edu

Department of Mathematics, University of Virginia, Charlottesville, VA 22903 